\title{
Hyperthermia and Thermosensitive Liposomes for Improved Delivery of Chemotherapeutic Drugs to Solid Tumors
}

\author{
Gerben A. Koning • Alexander M. M. Eggermont • Lars H. Lindner • Timo L. M. ten Hagen
}

Received: 4 December 2009 / Accepted: 8 April 2010 / Published online: 28 April 2010

(C) The Author(s) 2010. This article is published with open access at Springerlink.com

\begin{abstract}
Lipid-based nanocarriers or liposomes have been proven successful in the delivery of chemotherapeutic agents and are currently applied clinically in the treatment of various types of cancer. Liposomes offer the advantage of a high drug payload, decreased drug toxicity and enhanced drug accumulation at tumor sites. Increased accumulation is due to the relatively leaky tumor vasculature that allows liposome extravasation. Between different types of tumors and even within one tumor, vascular permeability and thus liposome extravasation may differ greatly. Furthermore, upon accumulation of liposomes in the tumor area, drug bioavailability is not guaranteed. At present, these are the major issues for clinically used liposomal drugs.

Mild hyperthermia $(H T)$, the heating of tumor tissue to temperatures of up to $43^{\circ} \mathrm{C}$, has been developed in the past decades as an established and efficacious treatment modality in combination with chemo- and radiotherapy. HT can be used to further improve liposomal chemotherapy in two ways: HT is known to increase vascular permeability in solid tumors and may therefore increase levels of liposome accumulation, and thermosensitive liposomes have been developed that can be triggered to release their contents upon hyperthermia. By applying these two strategies, drug delivery to tumors can be strongly enhanced.
\end{abstract}

\footnotetext{
G. A. Koning $(\varangle) \cdot$ A. M. M. Eggermont $\cdot$ L. H. Lindner •

T. L. M. ten Hagen

Laboratory Experimental Surgical Oncology, Section Surgical Oncology

Department of Surgery, Erasmus Medical Center, Rotterdam

PO Box 2040, 3000 CA Rotterdam, The Netherlands

e-mail: G.Koning@erasmusmc.nl

L. H. Lindner

Department of Internal Medicine III, University Hospital Grosshadern Ludwig-Maximilians University

Munich, Germany
}

KEY WORDS cancer - chemotherapy - hyperthermia . nanomedicine $\cdot$ thermosensitive liposomes · triggered release

\section{LIPOSOMAL DRUG DELIVERY}

In the past 40 years, liposomes, phospholipid-based nanovesicles, have been studied extensively as a drug delivery system, for example for the treatment of tumors. They currently represent one of the best studied types of drug delivery systems and are being used clinically either as experimental drug in various stages of clinical trials or as an approved and established drug. Examples of the latter are liposomal amphotericin B (Ambisome $\left.{ }^{\circledR}\right)$ for fungal infections, liposomal daunorubicin (Daunoxome $\left.{ }^{\circledR}\right)$, and pegylated liposomal doxorubicin (PLD) (Doxil ${ }^{\circledR} /$ Caelyx $\left.{ }^{\circledR}\right)$, both for anticancer therapy. Liposomes have attractive characteristics for use in drug delivery. Hydrophilic drugs can be entrapped in the liposome's aqueous compartments, while the lipid bilayer can be utilized to incorporate hydrophobic drugs. Associating a drug with liposomes will markedly change its pharmacokinetic properties and lower its systemic toxicity; furthermore, the drug is prevented from early inactivation in circulation. A major advancement in the use of liposomes was the development of small $(\sim 100 \mathrm{~nm})$ longcirculating liposomes, which renders the carrier and thus its drug contents with a long blood-residence time. These favorable circulation properties result in an enhanced accumulation of liposomal drugs in the tumor area (Fig. 1A) (1). Tumor sites are characterized by an immature leaky vasculature due to angiogenesis. However, the increased vascular permeability of tumor vasculature varies strongly between different tumor types and even within a tumor as was demonstrated in animal tumor models $(2,3)$ as well as in cancer patients (4). 

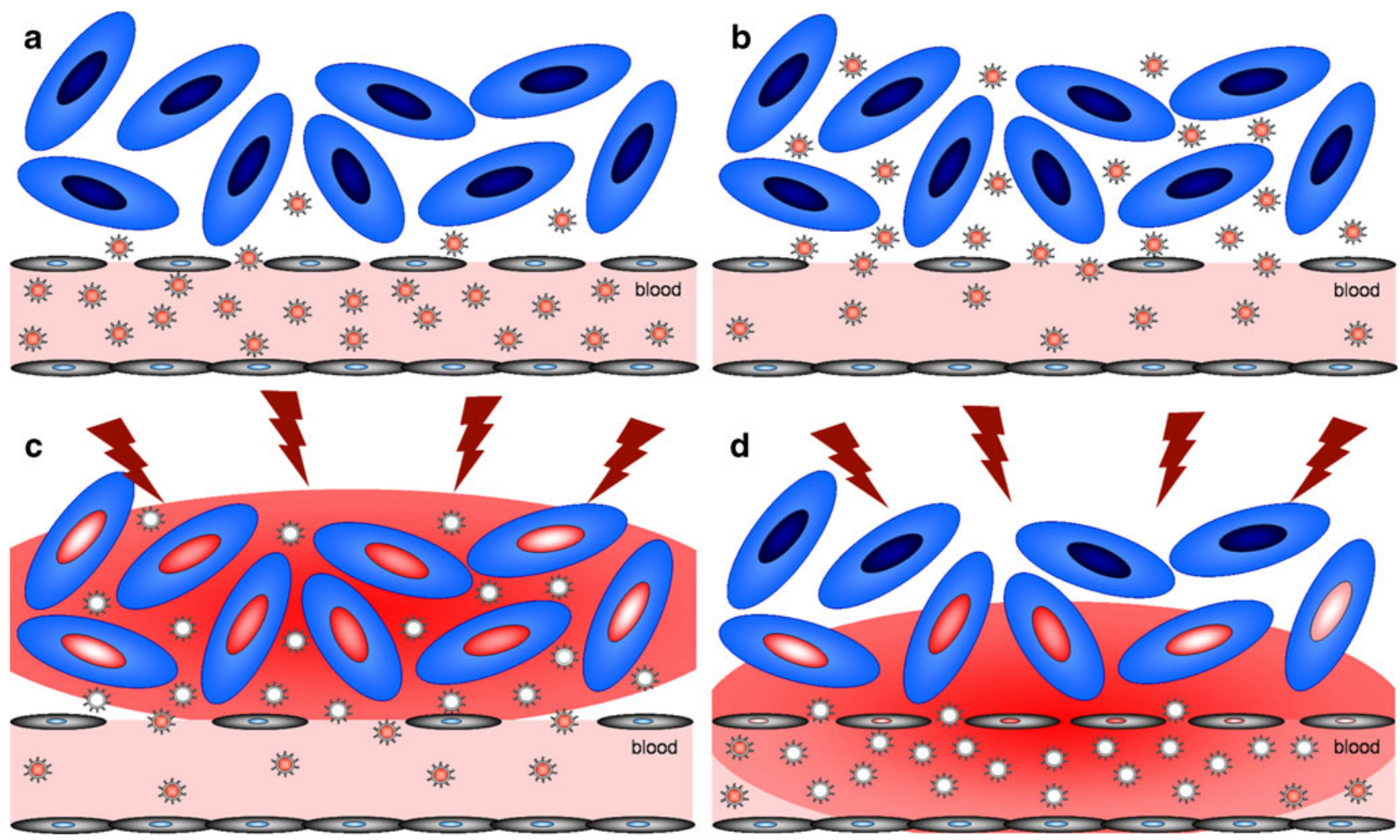

Fig. I Beneficial effects of hyperthermia on liposome extravasation and drug delivery to solid tumors. A. liposomes by virtue of their prolonged circulation capacity and small size may extravasate from tumor vasculature, which is more permeable than normal vasculature. B. Hyperthermia is known to increase vascular permeability, giving rise to increased levels of liposomes accumulating in the tumor tissue. $\mathbf{C}$. In the interstitial drug release approach, a heat trigger is applied to rapidly release drugs from tumor-localized thermosensitive liposomes in close proximity of tumor cells (represented by a red drug gradient in the tumor tissue). Subsequently, the drug is mainly taken up by tumor cells after which it can reach its site of action, e.g. the nucleus. D. In the intravascular release approach, thermosensitive liposomes reach the heated tumor area and immediately start releasing their drug contents intravascularly (represented by a red drug gradient arising from the tumor blood vessel). Released drugs will be absorbed by the tumor tissue and can be taken up by both tumor and endothelial cells.

Anthracyclines represent the main group of cytostatic drugs that have been associated with liposomes. Liposomal formulations of doxorubicin and daunorubicin are either approved or under clinical investigation for treatment of several types of cancer (5), e.g. Kaposi's Sarcoma, ovarian cancer, metastatic breast cancer, and multiple myeloma. A major benefits of the liposomal anthracyclin delivery compared to administration of the free drug is decreased (cardio)toxicity, with remaining efficacy also in typically anthracyclin-resistant tumors. However, in general, in all these clinical trials, no strong increases in therapeutic efficacy of liposomal doxorubicin have been described in comparison to the free drug. A main reason for this is that accumulation of liposomes in the tumor area does not guarantee that the encapsulated drug becomes bioavailable to the tumor cells. Not much is known about the actual fate of liposomal doxorubicin upon extravasation in a tumor. Seynhaeve et al. showed a significant level of uptake of liposomal doxorubicin by tumor cells followed by slow intracellular release, which ultimately renders the doxorubicin bioavailable as evidenced by its nuclear localization
2-3 days after administration (3). Laghina et al. quantified bioavailability of liposomal doxorubicin to be around 30 to $50 \%$ over several days after administration by measuring total tumor drug levels in comparison to the actual bioavailable drug levels measured in tumor cell nuclei (6).

Summarizing, results from clinical trials are promising but also leave room for further improvement. At least two main strategies are being recognized to this respect: 1) increased and more homogeneous accumulation of liposomal drugs in tumors and 2) increased bioavailability of the drugs by triggered release from liposomes to tumor cells. It is our strong belief that hyperthermia can be of great importance to achieve success in both suggested strategies.

\section{HYPERTHERMIA}

Mild hyperthermia involves the heating of tumors to temperatures of up to 43 degrees and is usually combined with radiation or chemotherapy to enhance the therapeutic outcome of these treatments. Presently, hyperthermia 
technology has been perfected and can be applied locally on the tumor by focusing electromagnetic or ultrasound energy on the tumor area using, e.g., radiofrequency electrodes implanted in the tumor or microwave antennas and ultrasound transducers that apply their energy to the tumor non-invasively.

Mild hyperthermia is known to cause major increases in tumor perfusion, this being one main reason for its synergistic effects with chemo and radiation treatment. In radiation treatment, increased perfusion will increase tumor oxygen levels and thereby the detrimental effects of the applied radiation. Improved drug delivery relies on the fact that local heating results in perforation of tumor blood vessels, microconvection in the interstitium, and perforation of the cancer cell membrane. Altogether, this enhances delivery of drugs from the blood into cancer cells with minimal thermal and mechanical damages to the normal tissues. In addition, hyperthermia can render tumor cells temporarily more sensitive to the damaging effects of radiation or chemotherapeutics. Another important effect of hyperthermia is DNA repair inhibition.

Firm clinical proof of increased efficacy upon combining hyperthermia with chemotherapy has been obtained in the past 10 years from a randomized trial in bladder cancer (7), a trial in cervical cancer (8) and, more recently, a large randomized EORTC trial in high-grade soft tissue sarcomas. Results of the latter study by Issels et al. were presented at the meeting of the European Cancer Organization 2009 in Berlin. In this study, the overall survival was strongly increased in the group of patients that completed the hyperthermia and chemotherapy treatment combination protocol compared to the group of patients receiving chemotherapy alone (9). Altogether, these data demonstrate that hyperthermia, after many years of development, has become an established treatment in cancer therapy with a high potency of improving the outcome of chemotherapy in various types of cancer.

\section{HYPERTHERMIA TO IMPROVE LIPOSOMAL CHEMOTHERAPY}

One of the next questions to be investigated is whether hyperthermia is able to increase the efficacy of liposomal chemotherapy. At present, there seems to be significant evidence in favor of such an approach. The remainder of this article will deal with these clinically relevant options.

In analogy to the successful combination of vasoactive compounds such as tumor necrosis factor alpha and liposomal chemotherapy to increase tumor drug levels and anti tumor activity $(3,10)$, hyperthermia can induce similar effects. Since hyperthermia has a major impact on several tumor pathophysiological parameters, including perfusion and vascular permeability, favorable effects on liposome extravasation in tumor areas, as represented schematically in Fig. 1B, can be anticipated and have already been demonstrated in some animal tumor models (11). The application of heat to tumors in animals in principle can involve similar technology as in humans, but is usually applied in a more straightforward way, for instance by submersing s.c. tumor-bearing extremities in a heating bath (12). Intravital microscopic imaging is a tool often used to visualize intratumoral fate of thermosensitive liposomes and release of their content. For this approach, heating of tissues placed in a dorsal skin-fold window chamber with implanted tumors can be achieved by placing it in a heated incubator (13), by using conductive heating for the whole (metal) window chamber (14), or, more precisely, by using a circular conductive heating ring designed for the window chamber (15).

More detailed studies during and after hyperthermia moving towards the involved vascular and cellular mechanisms, the actual fate of the liposomes after extravasation and the reproducibility of these effects in various animal tumor models and ultimately in cancer patients, are important items for the future.

\section{THERMOSENSITIVE LIPOSOMES}

Upon arrival in the tumor area, heat may also be applied to trigger the release of liposomal drug contents (Fig. 1C). To achieve this, thermosensitive liposomes are being developed. The formulation of such liposomes is based on pioneering work of Yatvin and Weinstein in the late 1970s (16), who described the use of liposomes composed of phospholipids that undergo a gel-to-liquid crystalline phase transition at temperatures of around $44^{\circ} \mathrm{C}$, a process which, in the absence of cholesterol in the membrane, causes significant release of liposome-entrapped watersoluble compounds.

From this basic formulation, thermosensitive liposomes have been further developed, for instance, by providing them with long-circulating properties using poly(ethylene glycol) $(12,15,17)$ or oligoglycerol-moieties (14) and by incorporating additional lipid compounds that further enhance membrane permeability at the phase transition temperature of the lipid membrane, e.g. lysolipid (12) or oligoglycerol-PG (14).

Importantly, the lysolipid formulation developed by Needham and Dewhirst's groups at Duke (USA) underwent further pharmaceutical development by the biopharmaceutical company Celsion and has now reached the clinical testing phase and is marketed as Thermodox ${ }^{\circledR}$. Two main studies are currently being performed combining Thermo- 
dox with hyperthermia in patients with loco-regional breast carcinoma of the chest wall and Thermodox with radiofrequency ablation in patients with primary or metastatic liver cancer. Results of these trials have not been published yet; however, they have already justified further clinical phase III testing in liver cancer patients (information obtained from the company's website at www.celsion.com).

These promising developments prove the high potential of combining hyperthermia with thermosensitive liposomes for delivery of chemotherapy to solid tumors.

\section{INTRAVASCULAR AND INTERSTITIAL DRUG RELEASE APPROACHES}

The lysolipid-based thermosensitive liposomes are injected just prior to or during the hyperthermia treatment with immediate release of their contents upon arrival in the heated tumor area, which is different from using the abovementioned long-circulating liposomal carriers for chemotherapy delivery to solid tumors. This approach is referred to as the intravascular drug release approach and skips the liposomal extravasation step to get into close proximity of the tumor cells (Fig. 1D). The released drug, currently doxorubicin, has been shown to be delivered efficiently from the tumor vasculature to the tumor cells as well as to vascular endothelial cells (18). More studies need to be done in order to establish the efficiency of this delivery process and whether this holds true for other chemotherapeutic drugs as well.

Besides the intravascular release approach, combinations of thermosensitive liposomes and hyperthermia are being developed that aim at interstitial release (Fig. 1G). In this approach, a primary hyperthermia treatment is applied to increase tumor vascular permeability, after which thermosensitive liposomes are administered that can extravasate into the tumor area. Exact and optimal requirements for the first hyperthermia treatment are not known. Kong et al. showed increasing levels of extravascular appearance of liposomes with increasing temperatures from 39 to $42^{\circ} \mathrm{C}$ applied for $60 \mathrm{~min}$ to a human ovarian carcinoma xenograft tumor in mice (11). They also demonstrated that the increased vascular permeability in this tumor model lasted up to $4 \mathrm{~h}$ after treatment. Upon reaching optimal liposome accumulation levels in the tumor, a second hyperthermia treatment can be applied to trigger drug release from the tumor-localized liposomes in very close proximity of the tumor cells. Since drug release from thermosensitive liposomes can occur very fast, with burst release of up to $70 \%$ of content in several minutes to maximal release within $1 \mathrm{~h}$ of hyperthermia $(12,14,15)$, the duration of the second heat trigger can be tailored accordingly.

\section{IMAGING-GUIDED DRUG DELIVERY}

Complicated drug delivery approaches as described above would certainly benefit from imaging guidance. Therefore, major efforts are being undertaken to provide liposomes with contrast agents to monitor tumor accumulation levels and drug release (1). Regarding thermosensitive liposomes, radionuclides and nuclear imaging can be used to image tumor accumulation of liposomes, but not drug release. Magnetic resonance imaging (MRI) to this respect has the advantage of being able to detect both liposome accumulation and drug release when a co-encapsulated MRI contrast agent is released together with the drug $(19,20)$. In addition, MRI thermometry information on tumor temperatures during hyperthermia is available online. Imaging-guided drug delivery using thermosensitive liposomal nanocarriers will ultimately help to further optimize and personalize cancer treatment and to get detailed online information on the applied treatment.

\section{CONCLUSION}

In conclusion, the combination of two separate developments in cancer therapy that have already been initiated in the 1970 s seem to have promising potential to contribute to further improvement of cancer therapy. The use of thermosensitive liposomes in combination with hyperthermia will help to further improve the delivery of chemotherapeutic drugs to solid tumors. The liposomes will retain their drug while in circulation, thereby preventing normal tissue exposure and related side effects. Upon arrival in the tumor area, they will only on command release their contents by the application of local hyperthermia, resulting in drug becoming bioavailable in close proximity of the target tumor cells. As a matter of fact, this approach is now undergoing clinical evaluation, which is a major step forward. Current and future developments in this area will include imaging-guided drug delivery approaches in which the whole treatment, including liposome accumulation, triggered drug release and tumor temperatures, can be monitored online. This will contribute to further improved efficacy of this combination treatment for the individual patient.

Open Access This article is distributed under the terms of the Creative Commons Attribution Noncommercial License which permits any noncommercial use, distribution, and reproduction in any medium, provided the original author(s) and source are credited. 


\section{REFERENCES}

1. Koning GA, Krijger GC. Targeted multifunctional lipid-based nanocarriers for image-guided drug delivery. Anticancer Agents Med Chem. 2007;7:425-40.

2. Dvorak HF, Nagy JA, Dvorak JT, Dvorak AM. Identification and characterization of the blood vessels of solid tumors that are leaky to circulating macromolecules. Am J Pathol. 1988;133:95-109.

3. Seynhaeve AL, Hoving S, Schipper D, Vermeulen CE, WielAmbagtsheer G, van Tiel ST et al. Tumor necrosis factor alpha mediates homogeneous distribution of liposomes in murine melanoma that contributes to a better tumor response. Cancer Res. 2007;67:9455-62.

4. Harrington KJ, Mohammadtaghi S, Uster PS, Glass D, Peters AM, Vile RG et al. Effective targeting of solid tumors in patients with locally advanced cancers by radiolabeled pegylated liposomes. Clin Cancer Res. 2001;7:243-54.

5. Alberts DS, Muggia FM, Carmichael J, Winer EP, Jahanzeb M, Venook AP et al. Efficacy and safety of liposomal anthracyclines in phase I/II clinical trials. Semin Oncol. 2004;31:53-90.

6. Laginha KM, Verwoert S, Charrois GJ, Allen TM. Determination of doxorubicin levels in whole tumor and tumor nuclei in murine breast cancer tumors. Clin Cancer Res. 2005;11:6944-9.

7. Colombo R, Da Pozzo LF, Salonia A, Rigatti P, Leib Z, Baniel J et al. Multicentric study comparing intravesical chemotherapy alone and with local microwave hyperthermia for prophylaxis of recurrence of superficial transitional cell carcinoma. J Clin Oncol. 2003;21:4270-6.

8. Franckena M, De Wit R, Ansink AC, Notenboom A, Canters RA, Fatehi D et al. Weekly systemic cisplatin plus locoregional hyperthermia: an effective treatment for patients with recurrent cervical carcinoma in a previously irradiated area. Int J Hyperthermia. 2007;23:443-50.

9. Issels R, Lindner LH, Wendtner CM, Hohenberger P, Reichardt $\mathrm{P}$, Daugaard S et al. Impact of regional hyperthermia (RHT) on response to neo-adjuvant chemotherapy and survival of patients with high-risk soft-tissue sarcoma (HR-STS): Results of the randomized EORTG-ESHO intergroup trial (NCI-00003052). Eur J Cancer Suppl. 2009;7:2-3.
10. ten Hagen TL, Van Der Veen AH, Nooijen PT, van Tiel ST, Seynhaeve AL, Eggermont AM. Low-dose tumor necrosis factor-alpha augments antitumor activity of stealth liposomal doxorubicin (DOXIL) in soft tissue sarcoma-bearing rats. Int J Cancer. 2000;87:829-37.

11. Kong G, Braun RD, Dewhirst MW. Characterization of the effect of hyperthermia on nanoparticle extravasation from tumor vasculature. Cancer Res. 2001;61:3027-32.

12. Needham D, Anyarambhatla G, Kong G, Dewhirst MW. A new temperature-sensitive liposome for use with mild hyperthermia: characterization and testing in a human tumor xenograft model. Cancer Res. 2000;60:1197-201.

13. Kong G, Braun RD, Dewhirst MW. Hyperthermia enables tumor-specific nanoparticle delivery: effect of particle size. Cancer Res. 2000;60:4440-5.

14. Lindner LH, Eichhorn ME, Eibl H, Teichert N, Schmitt-Sody M, Issels RD et al. Novel temperature-sensitive liposomes with prolonged circulation time. Clin Cancer Res. 2004;10:2168-78.

15. Li L, ten Hagen TL, Schipper D, Wijnberg TM, Van Rhoon GC, Eggermont AM et al. Triggered content release from optimized stealth thermosensitive liposomes using mild hyperthermia. J Control Release. 2010;143:274-9.

16. Yatvin MB, Weinstein JN, Dennis WH, Blumenthal R. Design of liposomes for enhanced local release of drugs by hyperthermia. Science. 1978;202:1290-3.

17. Unezaki S, Maruyama K, Takahashi N, Koyama M, Yuda T, Suginaka A et al. Enhanced delivery and antitumor activity of doxorubicin using long-circulating thermosensitive liposomes containing amphipathic polyethylene glycol in combination with local hyperthermia. Pharm Res. 1994;11:1180-5.

18. Chen Q Tong S, Dewhirst MW, Yuan F. Targeting tumor microvessels using doxorubicin encapsulated in a novel thermosensitive liposome. Mol Cancer Ther. 2004;3:1311-7.

19. Ponce AM, Viglianti BL, Yu D, Yarmolenko PS, Michelich CR, Woo $\mathrm{J}$ et al. Magnetic resonance imaging of temperature-sensitive liposome release: drug dose painting and antitumor effects. J Natl Cancer Inst. 2007;99:53-63.

20. Peller M, Schwerdt A, Hossann M, Reinl HM, Wang T, Sourbron $\mathrm{S}$ et al. MR characterization of mild hyperthermiainduced gadodiamide release from thermosensitive liposomes in solid tumors. Invest Radiol. 2008;43:877-92. 